\title{
Sodium Alginate
}

National Cancer Institute

\section{Source}

National Cancer Institute. Sodium Alginate. NCI Thesaurus. Code C84148.

The sodium salt form of alginic acid and gum mainly extracted from the cell walls of brown algae, with chelating activity. Upon oral administration, sodium alginate binds to and blocks the intestinal absorption of various radioactive isotopes, such as radium $\mathrm{Ra}$ 226 (Ra-226) and strontium Sr 90 (Sr-90). 